\title{
Neue Arten der Gattung Mescirtes Motschulsky, 1863 aus Laos und Vietnam (Coleoptera, Scirtidae) (190. Beitrag zur Kenntnis der Scirtidae)
}

\author{
Mit 20 Figuren und 2 Tabellen \\ BERNHARD KLAUSNITZER ${ }^{1}$ \\ ${ }^{1}$ Mitglied des Senckenberg Deutschen Entomologischen Instituts, Lannerstraße 5, 01219 Dresden, Deutschland \\ Published on 2013-12-20
}

\section{Zusammenfassung}

Aus Laos bzw. Vietnam wird je eine neue Art aus der Gattung Mescirtes Motschulsky, 1863 beschrieben. M. sedlagi n. sp. ist vor allem durch die auffällige Färbung gekennzeichnet. M. eberti n. sp. wird mit M. rutilicollis KLAUSNITzER, 2012 verglichen. Bestimmungstabellen für die Männchen und Weibchen der aus Südostasien (Festland) bekannten Arten sowie ein Katalog aller Mescirtes werden vorgelegt.

\section{Summary}

One new species of Mescirtes Motschulsky, 1863 from Laos and a second from Vietnam are described. M. sedlagi n. sp. is mainly characterized by its distinctive colouration. M. eberti n. sp. is compared with M. rutilicollis KLAUsNITZER, 2012. Identification keys for males and females of the species known from Southeast Asia (mainland) and a catalog of all Mescirtes are presented.

\section{Key words}

Coleoptera, Scirtidae, Mescirtes, new species, determination key, Laos, Vietnam, Oriental Region.

\section{Einleitung}

Arten der Gattung Mescirtes Motschulsky, 1863 sind in der Orientalischen Region weit verbreitet (siehe 6.) (Klausnitzer 2005, 2009a, b, 2010, 2012a, b, 2013, Ruta 2009, 2010, Yoshiтomi \& SAtô 2003), kommen aber auch in der Äthiopischen Region vor (Ruta 2010). Auch aus Nepal sind zwei Arten bekannt. In der vorliegenden Arbeit können zwei Arten neu beschrieben werden.

\section{Beschreibung von Mescirtes sedlagi n. sp.}

Holotypus: $\sigma^{\star}$, Laos, Xieng Khouang Prov., $19^{\circ} 37-8^{\prime} \mathrm{N}$, $103^{\circ} 20-1 E$, Phonsavan (30 km NE): Phou Sane Mt., 14001700 m, 10.-30.5.2009, D. Hauck leg. / NHMB Basel, NMPC Prague Laos 2009 Expedition, M. Brancucci, M. Geiser, Z. Kraus, D. Hauck, V. Kubáň. In coll. Naturhistorisches Museum Basel.

Paratypus: + , Etikettierung wie Holotypus. In coll. B. Klausnitzer. 
Körperlänge (Länge Pronotum + Elytre): 3,18 $\left(\sigma^{\star}\right)$, 2,49 ( 9 ) mm. Körper rundlich (Fig. 1,2): Verhältnis

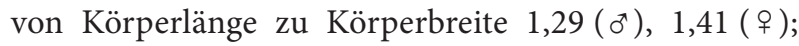
gewölbt: Höhe der Elytren in Seitenansicht 0,95 ( $\left.\sigma^{*}\right)$, 0,82 (ㅇ) $\mathrm{mm}$.

Kopf dunkel rotbraun, nach vorn heller rotbraun; dicht weiß behaart; fein punktiert. Kopf zwischen den Augen 0,75 $\left(\mathrm{o}^{\star}\right), 0,65$ ( + ) $\mathrm{mm}$ breit. Clypeus ausgerandet, Vorderecken nach vorn gezogen, dazwischen gerundet eingebuchtet. Mandibeln braun. Labrum braun, Seitenrand gebogen, Vorderrand etwas eingebuchtet. 1. Antennenglied braun, übrige Antennenglieder hellbraun. Maße siehe Tabelle 1.

Pronotum schwarzbraun, Seitenrand schmal aufgehellt (Fig. 1,2); fein punktiert, Punktzwischenräume glatt (100fache Vergrößerung), weiß behaart; Vorderecken etwas vorgezogen. Maximale Breite 1,72 $\left(\sigma^{*}\right)$, 1,07 ( ㅇ ) mm; Länge in der Mitte 0,67 ( $\left.0^{*}\right), 0,52$ (ㅇ $) \mathrm{mm}$. Scutellum dunkel rotbraun, fein punktiert, hell behaart.

Elytren schwarzbraun, in der Mitte befindet sich ein gemeinsamer scharf begrenzter tropfenförmiger orangenfarbiger Fleck (Fig. 1,2) von 1,95 mm Länge und einer maximalen Breite von 1,40 mm ( $\left.\star^{\star}\right)$, der nach hinten schmaler wird. Elytren kräftiger als das Pronotum punktiert, Punktzwischenräume glatt (100fache Vergrößerung); weiß behaart. Breite einer Elytre in der Mitte

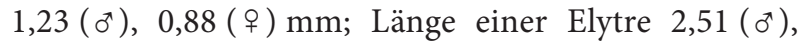
1,97 (우) $\mathrm{mm}$.

Beine braun. Hinterbein $o^{\star}$ : Länge der Tibia 0,91 mm; großer Sporn schwach gebogen, innen etwas gekerbt, spitz endend, ohne Berücksichtigung der Krümmung 0,08 mm lang; 1 . Tarsenglied 0,17 mm lang. 3. bis 7. Sternit rotbraun, Ränder dunkelbraun $\left(\sigma^{\star}\right)$, bzw. hellbraun, Ränder braun ( 9 ).

\section{Männchen}

7. Sternit hinten schwach eingebuchtet (Buchttiefe 0,02 mm); maximale Länge neben der Bucht 0,30 mm; maximale Breite $1,13 \mathrm{~mm}$.

8. Sternit im Umriss dreieckig, eine schmale bogenförmige Spange bildend (Fig. 4), die eine dünne Membran umfasst. Länge ca. 0,25 mm; Breite hinten 0,30 mm.

Platte des 9. Sternit in zwei Lappen geteilt, die hinten mit wenigen Borsten besetzt sind (Fig. 5), vorn ist die Platte breit abgestutzt. Jederseits befindet sich eine schwach entwickelte, unscharf begrenzte schmale Sklerotisierung. Maximale Länge 0,32 $\mathrm{mm}$; Breite hinten 0,27 mm.
7. Tergit (Fig. 6) aus einer breiten, hinten schwach gebogenen Platte und etwas gekrümmten Bacilla lateralia bestehend. Der Hinterrand ist mit einem dichten Saum feiner spitzer Haare bedeckt. Gesamtlänge $0,40 \mathrm{~mm}$; maximale Breite der Platte ca. 0,80 mm; Länge der Bacilla lateralia ohne Berücksichtigung der Krümmung 0,31 mm.

8. Tergit (Fig. 7) aus einer breiten, hinten eingebogenen Platte (Tiefe der Bucht 0,04 mm) und kräftig sklerotisierten gekrümmten Bacilla lateralia bestehend. Die Platte ist mit kurzen Borsten besetzt. Gesamtlänge $0,40 \mathrm{~mm}$; Breite der Platte $0,50 \mathrm{~mm}$; Länge der Bacilla lateralia ohne Berücksichtigung der Krümmung 0,33 mm.

Platte des 9. Tergit breit, mit etwas ausgebogenen Seiten (Fig. 8) In der Mitte befindet sich eine transverse Sklerotisierung (eventuell ein Präparationsartefakt und durch Umschlagen eines Teils der Platte entstanden). Bacilla lateralia nur wenig gebogen. Gesamtlänge $0,40 \mathrm{~mm}$; Breite der Platte 0,28 mm; Länge der Bacilla lateralia ohne Berücksichtigung der Krümmung 0,26 mm.

Tegmen (Fig. 9) aus einer breiten, vorn zugespitzten, nach hinten breiter werdenden Platte bestehend. Die Parameren sind breit, fast parallel und enden gerundet. Tegmen an den Seiten und die Parameren bis kurz vor der Spitze mit Sinnesporen und kleinen Borsten besetzt. Gesamtlänge 0,57 mm; maximale Breite 0,26 mm.

Penis asymmetrisch, Pala länglich oval und an den Seiten verstärkt (Fig. 10), Vorderrand gleichmäßig gebogen. Trigonium sklerotisiert (Fig. 11), innen in zwei aufeinander gerichtete Spitzen auslaufend (Pfeil), hinten etwas eingebuchtet. Parameroide lang, weitgehend voneinander getrennt. Gesamtlänge des Penis 0,56 mm; Breite der Pala $0,12 \mathrm{~mm}$; Länge der Pala bis zur Spitze des Trigonium 0,49 mm; Länge der Parameroide ab Pala 0,11 mm; Länge des Trigonium $0,04 \mathrm{~mm}$.

\section{Weibchen}

Hinterrand des 7. Sternit gleichmäßig gebogen, im Mittelbereich etwas abgestutzt, in der Mitte 0,29 mm lang; maximal 0,94 $\mathrm{mm}$ breit.

7. Tergit aus einer breiten, hinten gebogenen Platte und schwach gekrümmten Bacilla lateralia bestehend. Der Hinterrand ist mit einem dichten Saum feiner spitzer Haare bedeckt. Maximale Breite der Platte 0,62 mm; Länge der Platte in der Mitte $0,32 \mathrm{~mm}$; Länge der Bacilla lateralia ohne Berücksichtigung der Krümmung $0,36 \mathrm{~mm}$.

Das 8. Sternit bildet eine geschlossene Platte. Neben den Seitenrändern befindet sich jederseits fast auf der gesam- 
ten Länge ein sklerotisiertes Stäbchen. Gesamtlänge des 8. Sternit 0,56 mm; maximale Breite $0,26 \mathrm{~mm}$.

8. Tergit mit einer kurzen, fast quadratischen Platte, die an der Spitze mit Mikrotrichen besetzt ist und langen Bacilla lateralia. Gesamtlänge 0,98 mm; maximale Breite der Platte $0,24 \mathrm{~mm}$.

Ovipositor dem Grundbauplan der Gattung entsprechend. Styli 0,06 mm lang; Länge Coxite 0,23 mm; Länge Baculi 1,27 mm; Gesamtlänge 1,59 mm.

Das Bursalsklerit (Fig. 12) ist kräftig sklerotisiert und läuft hinten in drei Fortsätze aus. Die beiden seitlichen Arme

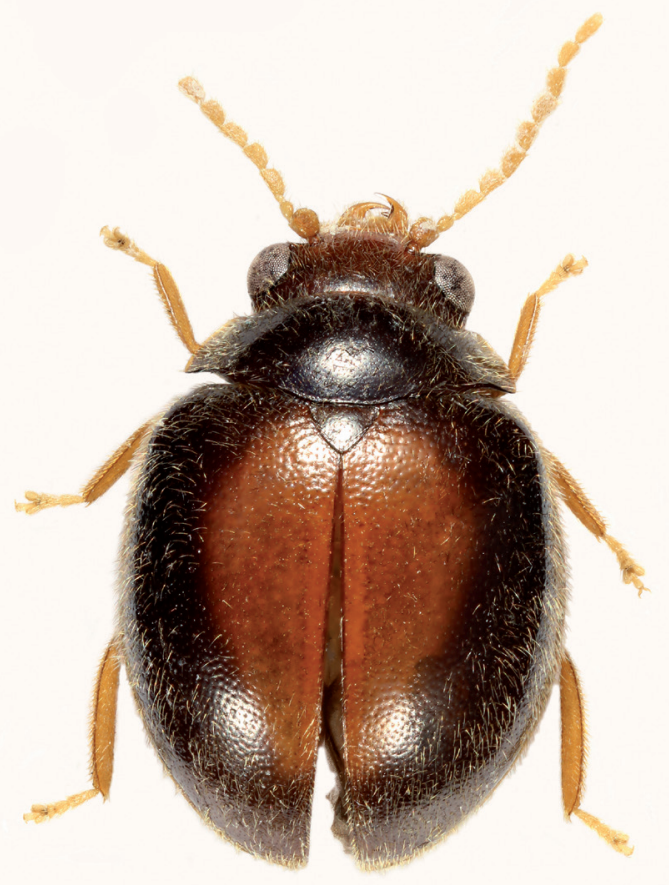

1 enden lappenförmig $(0,11 \mathrm{~mm}$ lang), der mittlere ist zahnförmig zugespitzt und trägt an der Spitze einen abgesetzten Zahn. Länge des Mittelfortsatzes von der Basis bis zur Spitze 0,12 mm. An der Hinterkante des Bursalsklerit befindet sich eine wellenförmige Skulptur. Länge des Bursalsklerit gesamt 0,22 mm; maximale Breite 0,22 mm.

Areal: Bisher nur vom locus typicus bekannt.

Derivatio nominis: Die neue Art widme ich meinem Lehrer, Herrn Prof. Dr. habil. Ulrich Sedlag, Eberswalde, anlässlich seines 90 . Geburtstages mit herzlichem Dank für sein stetes Interesse an meinen entomologischen Studien und für seine Unterstützung.

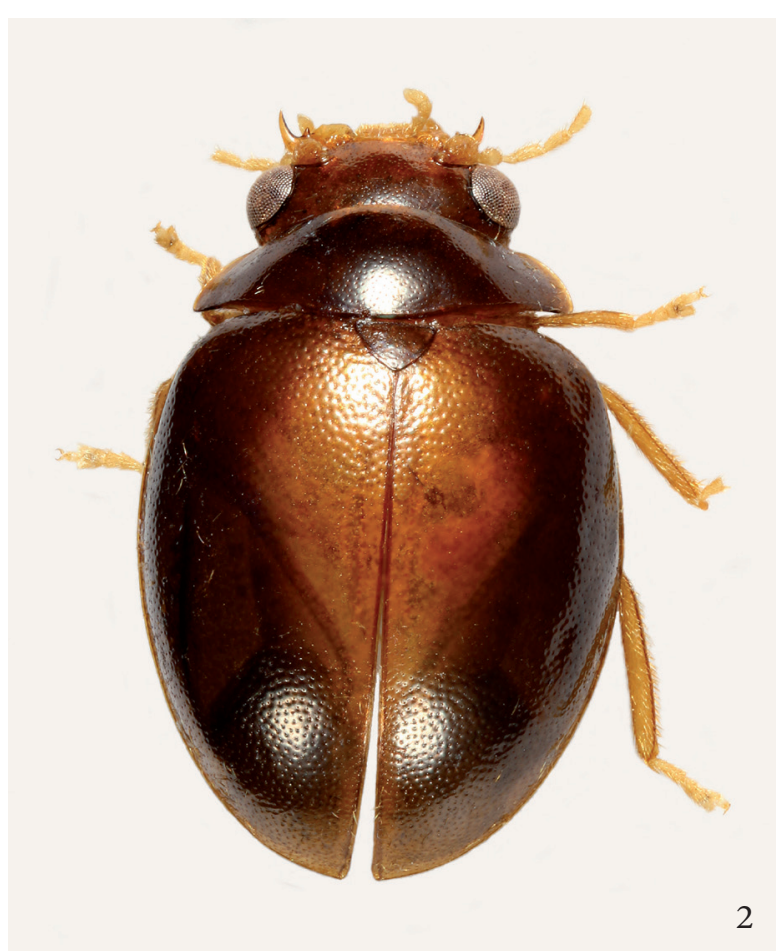

Fig. 1-2: Mescirtes sedlagi n. sp., (1 links) $\diamond^{\star}$, Habitus, dorsal (2 rechts) $\odot$, Habitus, dorsal.

Tabelle 1: Maße [mm] der Antennenglieder von Mescirtes sedlagi $\mathrm{n} . \mathrm{sp} . \mathrm{AG}=$ Antennenglied.

\begin{tabular}{|c|c|c|c|c|c|}
\hline $\mathrm{AG}$ & đ Länge & $\sigma^{\top}$ Breite & ㅇ Länge & ㅇ Breite & Form \\
\hline 1. & 0,17 & 0,15 & 0,14 & 0,11 & innen mit scharfer gebogener Kante \\
\hline 2. & 0,10 & 0,07 & 0,08 & 0,05 & zylindrisch \\
\hline 3. & 0,06 & 0,04 & 0,05 & 0,03 & zylindrisch \\
\hline 4. & 0,17 & 0,08 & 0,12 & 0,05 & $\begin{array}{l}\text { Außenseite gerade, beim }{ }^{\star} \text { etwas nach innen ausgebogen, abge- } \\
\text { flacht, beim }+ \text { annähernd zylindrisch }\end{array}$ \\
\hline 5. & 0,14 & 0,09 & 0,11 & 0,05 & wie 4. \\
\hline 6. & 0,16 & 0,07 & 0,12 & 0,04 & wie 4. \\
\hline 7. & 0,15 & 0,08 & 0,12 & 0,04 & wie 4. \\
\hline 8. & 0,15 & 0,08 & - & - & wie 4. \\
\hline 9. & 0,15 & 0,08 & - & - & zylindrisch \\
\hline 10. & 0,14 & 0,07 & - & - & zylindrisch \\
\hline 11. & 0,19 & 0,07 & - & - & zylindrisch \\
\hline
\end{tabular}




\section{Beschreibung von Mescirtes eberti n. sp.}

Holotypus: $\sigma^{\star}$, Vietnam-N, Quang Bien prov., $1 \mathrm{~km} \mathrm{~N}$ of Cha Lo, $400 \mathrm{~m}$, Vietnam-Laos border Area, 17²41'22"N, $105^{\circ} 45^{\prime} 45^{\prime \prime E}$, 11.-24.4.2010, L. Dembicky leg. In coll. Naturhistorisches Museum Basel.

Körperlänge (Länge Pronotum + Elytre): 2,53 mm. Körper rundlich (Fig. 3): Verhältnis von Körperlänge zu Körperbreite 1,23; gewölbt: Höhe der Elytren in Seitenansicht $0,98 \mathrm{~mm}$.

Kopf rotbraun, dicht weiß behaart, fein punktiert. Kopf zwischen den Augen 0,74 mm breit. Clypeus ausgerandet, Vorderecken nach vorn gezogen, dazwischen gerundet. Mandibeln braun. Labrum hellbraun, Seitenrand gebogen, Vorderrand eingebuchtet. 1. Antennenglied braun, übrige Antennenglieder gelbbraun. Maße siehe Tabelle 2 .

Pronotum rotbraun (Fig. 3), fein punktiert, Punktzwischenräume glatt (100fache Vergrößerung), weiß behaart, Vorderecken etwas vorgezogen. Maximale Breite $1,50 \mathrm{~mm}$; Länge in der Mitte $0,56 \mathrm{~mm}$. Scutellum rotbraun, fein punktiert, hell behaart.

Elytren schwarzbraun, Seitenrand hinten und Apex rotbraun (Fig. 3). Elytren kräftiger als das Pronotum punktiert, Punktzwischenräume glatt (100fache Vergrößerung), weiß behaart. Breite einer Elytre in der Mitte 1,03 mm; Länge einer Elytre 1,97 mm.

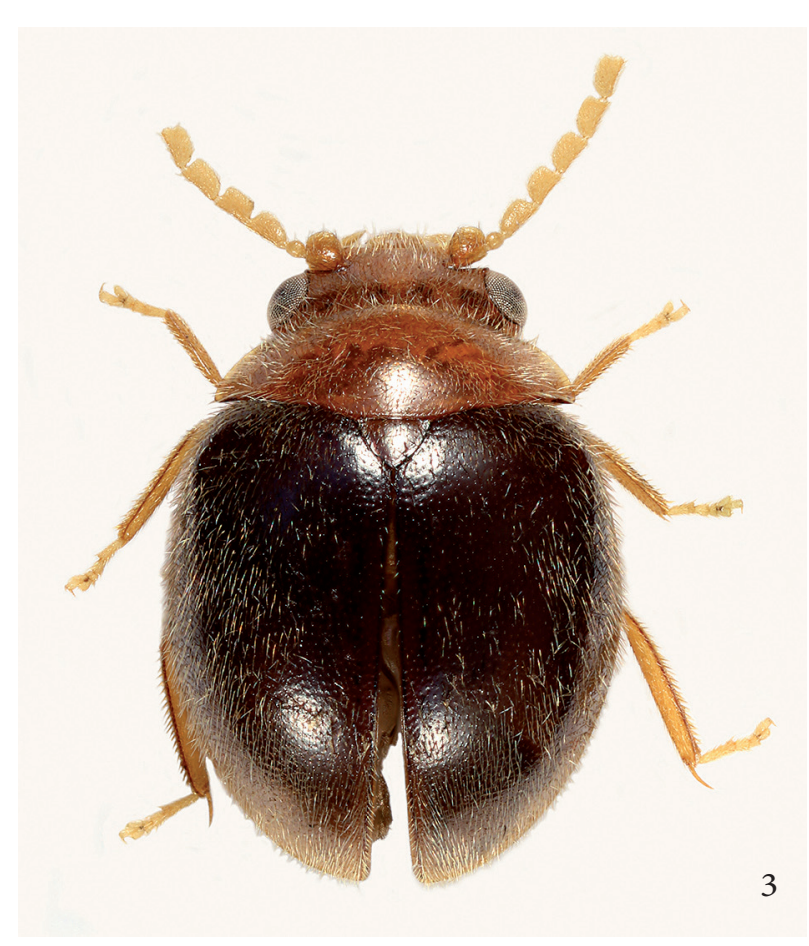

Fig. 3: Mescirtes eberti n. sp., ơ , Habitus, dorsal.
Beine braun. Hinterbein: Länge der Tibia 0,76 mm; großer Sporn schlank, schwach gebogen, spitz endend, ohne Berücksichtigung der Krümmung 0,16 mm lang; 1. Tarsenglied 0,18 mm lang. 3. bis 7. Sternit rotbraun, Ränder dunkelbraun.

7. Sternit hinten sehr schwach eingebuchtet (Buchttiefe 0,02 mm). Maximale Länge neben der Bucht 0,24 mm; maximale Breite $0,95 \mathrm{~mm}$.

8. Sternit eine schmale, längliche, bogenförmige Spange bildend (Fig. 13). Länge 0,26 mm; Breite hinten 0,17 mm.

Platte des 9. Sternit hinten in zwei zugespitzte Lappen geteilt, die je ca. 15 Borsten tragen (Fig. 14). An den etwas abgesetzten Innenkanten stehen die Borsten in einer Zeile. In der Mitte der Hemisternite befindet sich jederseits eine schmale Sklerotisierung. Länge 0,28 mm (vermutlich fehlt der Basalabschnitt); Breite hinten 0,32 mm.

7. Tergit (Fig. 15) aus einer breiten, hinten gebogenen Platte und schwach gekrümmten Bacilla lateralia bestehend. Der Hinterrand ist mit einem dichten Saum feiner spitzer Mikrotrichen bedeckt. Davor befindet sich eine schmale Zone mit paarig angeordneten Mikrotrichen. Gesamtlänge 0,43 mm; maximale Breite der Platte ca. 0,80 mm; Länge der Bacilla lateralia ohne Berücksichtigung der Krümmung 0,34 mm.

8. Tergit (Fig. 16) aus einer breiten, nach hinten verschmälerten und etwas abgestutzten Platte und gekrümmten Bacilla lateralia bestehend. Gesamtlänge $0,46 \mathrm{~mm}$; Breite der Platte 0,45 mm; Länge der Bacilla lateralia ohne Berücksichtigung der Krümmung 0,35 mm.

Platte des 9. Tergit schlank, mit annähernd parallelen Seiten (Fig. 17). Bacilla lateralia fast gerade. Gesamtlänge 0,50 mm; Breite der Platte ca. 0,28 mm; Länge der Bacilla lateralia $0,30 \mathrm{~mm}$.

Tabelle 2: Maße [mm] der Antennenglieder von Mescirtes eberti n. sp. AG = Antennenglied. 9.-11. Glied nicht erhalten.

\begin{tabular}{|lccl|}
\hline AG & $\sigma^{\star}$ Länge & $\sigma^{\star}$ Breite & Form \\
\hline 1. & 0,16 & 0,15 & $\begin{array}{l}\text { innen mit scharfer } \\
\text { gebogener Kante }\end{array}$ \\
2. & 0,08 & 0,07 & fast kugelförmig \\
3. & 0,05 & 0,03 & zylindrisch, dreieckig \\
4. & 0,19 & 0,09 & $\begin{array}{l}\text { Außenseite gerade, } \\
\text { nach innen etwas aus- } \\
\text { gebogen, abgeflacht }\end{array}$ \\
5. & 0,15 & 0,11 & wie 4. \\
6. & 0,18 & 0,10 & wie 4. \\
7. & 0,18 & 0,10 & wie 4. \\
8. & 0,17 & 0,09 & wie 4. \\
\hline
\end{tabular}


Tegmen (Fig. 18) aus einer breiten, vorn abgestutzten, fast quadratischen Platte bestehend. Die Parameren sind schlank, bis zur Mitte parallel, verschmälern sich dann abrupt, der schmale, ebenfalls parallele Distalabschnitt endet gerundet. Parameren mit einzelnen Borsten, an der Spitze mit Sinnesporen. Gesamtlänge $0,73 \mathrm{~mm}$; maximale Breite 0,40 mm; Breite an der Basis 0,20 mm; Länge der Parameren 0,53 mm.

Penis asymmetrisch, Pala länglich oval und an den Seiten verstärkt (Fig. 19), Vorderrand gleichmäßig gebogen. Trigonium gerundet, gespalten, kräftig sklerotisiert (Fig. 20), hinten mit etwas spitz endenden Schuppen bedeckt (Pfeil). Parameroide lang, weitgehend voneinander getrennt. Gesamtlänge des Penis $0,68 \mathrm{~mm}$; Länge der Pala bis zum Trigonium 0,48 $\mathrm{mm}$.
Weibchen: unbekannt.

Areal: Bisher nur vom locus typicus bekannt.

Derivatio nominis: Ich möchte die neue Art Herrn Prof. Dr. Werner Ebert, Eberswalde, zu seinem 85. Geburtstag widmen. Vor fast 60 Jahren begann unsere Freundschaft, bald folgten die Vorbereitungen zur Herausgabe des „Nachrichtenblatt der Oberlausitzer Insektenfreunde“, das bis heute in den „Entomologischen Nachrichten und Berichten" fortbesteht.

\section{Differentialdiagnose und Bestimmungstabelle für die in Hinterindien vorkommenden, als ơ o bekannten Arten von Mescirtes MotsCHULSkY, 1863}

Bestimmungstabellen aller bisher beschriebenen Arten finden sich bei KLAusnitzer (2012a, b, 2013). M. sedlagi n. sp. ist vor allem durch die Färbung unverwechselbar. M. eberti n. sp. kann von M. rutilicollis KLAUSNITZER, 2012 deutlich abgegrenzt werden.

1 Körper einfarbig rotbraun bis schwarzbraun. Differenzierung nach KLAUSNitzer (2012a, 2013). armatolaosensis KLAUSNITZER, 2012 (Laos) hansmalickyi Klausnitzer, 2010 (Vietnam, Laos) laosensis (Yoshiтomi Eт SATô, 2003) (Laos, Thailand) squamatilis KLAUSNItzer, 2013 (China, Yunnan)

$1^{\star}$ Elytren mit abgegrenzten Flecken oder Punkten, einem gemeinsamen hellen Fleck in der Mitte oder Pronotum und Elytren sind verschieden gefärbt.

2 Elytren mit abgegrenzten Flecken oder Punkten oder einem gemeinsamen hellen Fleck in der Mitte.

$2^{*}$ Elytren einfarbig, Pronotum und Elytren sind verschieden gefärbt (Pronotum rotbraun, Elytren schwarzbraun).

Elytren mit deutlich abgegrenzten Flecken oder Punkten. Differenzierung nach KLAusnitzer (2012a). herthae (KLAUSNITZER, 2009) (Laos) herthaelisae KLAUSNITZER, 2012 (Thailand)

$3^{*}$ Elytren schwarzbraun, in der Mitte mit einem gemeinsamen, scharf begrenzten, tropfenförmigen orangenfarbigen Fleck (Fig. 1). Körperlänge 3,18 mm. Laos. sedlagi n. sp.

4 Trigonium gerundet und kräftig sklerotisiert, hinten mit Körnchen dicht bedeckt. Parameren gleichmäßig nach hinten verjüngt. Scutellum schwarz, ähnlich wie die Elytren gefärbt. Körperlänge 2,51 mm. Thailand.

rutilicollis KLAUSNITZER, 2012

$4^{\star}$ Trigonium gespalten, mit spitz endenden Schuppen bedeckt (Fig. 20). Parameren etwa ab der Mitte abrupt verschmälert (Fig. 18). Scutellum rotbraun, ähnlich wie das Pronotum gefärbt. Körperlänge 2,53 mm. Vietnam. eberti n. sp. 


\section{Differentialdiagnose und Bestimmungstabelle für die in Hinterindien vorkommenden, als ㅇ o bekannten Arten von Mescirtes MotschULSKY, 1863}

Bestimmungstabellen aller bisher beschriebenen Arten finden sich bei KLAUSNitzer (2012b). Bisher sind nur von sieben Arten der Gattung Mescirtes die Weibchen bekannt (im Katalog mit * gekennzeichnet). Nur von M. herthae, M. sedlagi n. sp. und M. yoshitomii sind beide Geschlechter beschrieben. M. sedlagi n. sp. ist bereits durch die Färbung von den anderen Arten abzugrenzen. Hinzu kommt der charakteristische Bau des Bursalsklerit.

1 Elytren einfarbig. Mittelzahn des Bursalsklerit ungeteilt. Körperlänge 3,42 mm. Thailand.

brancuccii Klausnitzer, 2012

$1^{\star}$ Elytren gelbbraun bis braun, mit abgegrenzten dunklen Punkten. Mittelzahn des Bursalsklerit dreigeteilt. Differenzierung nach KLAUSNITZER (2012b). gudrunae Klausnitzer, 2010 (Vietnam) herthae (KLAUSNITZER, 2009) (Laos)

$1^{\star *}$ Elytren schwarzbraun, in der Mitte mit einem gemeinsamen, scharf begrenzten tropfenförmigen orangenfarbigen Fleck (Fig. 2). Mittelzahn des Bursalsklerit ungeteilt, mit einem abgesetzten Endzahn (Fig. 12). Körperlänge 2,49 mm. Laos. sedlagi n. sp.

\section{Katalog der Arten}

Mescirtes armatolaosensis KLAUSNITZER, 2012 - Laos ${ }^{*}$ Mescirtes brancuccii KLAUSNITZER, 2012 - Thailand Mescirtes eberti n. sp. - Vietnam

Mescirtes gagatinus Motschulsky, 1863 - Sri Lanka

${ }^{*}$ Mescirtes gudrunae Klausnitzer, 2010 - Vietnam

Mescirtes hansmalickyi KLAUSNITZER, 2010 - Vietnam, Laos

${ }^{*}$ Mescirtes herthae (KLAusnitzer, 2009) - Laos

Mescirtes herthaelisae KLAUSNITZER, 2012 - Thailand

Mescirtes laosensis (Yoshitomi ET SATô, 2003) - Laos, Thailand

Mescirtes rutai (Klausnitzer, 2009) - Malaysia; Pahang ${ }^{*}$ Mescirtes ruthsteuerae (KLAusnitzer, 2009) - Nepal Mescirtes rutilicollis KLAUSNITZER, 2012 - Thailand

${ }^{*}$ Mescirtes sedlagi n. sp. - Laos

Mescirtes squamatilis KLAusnitzer, 2013 - China (Yunnan)

${ }^{*}$ Mescirtes weigeli (KLAusnitzer, 2009) - Nepal

${ }^{*}$ Mescirtes yoshitomii (KLAusnitzer, 2009) - Sumatra

\section{Dank}

Herrn Dr. Michel Brancucci $\dagger$, Naturhistorisches Museum Basel, danke ich sehr herzlich für die stets großzügigen Möglichkeiten zur Bearbeitung der Scirtidae aus den Sammlungen des Museums. Die Abbildungen 1-3 fertigte freundlicherweise Herr Lutz Behne, Senckenberg Deutsches Entomologisches Institut Müncheberg an. Herrn Dr. Lothar Zerche vom gleichen Institut danke ich für Hinweise zum Manuskript.

\section{Literatur}

Klausnitzer, B. 2005: Eine neue Art der Gattung Scirtes Illiger, 1807 aus Thailand (Col., Scirtidae). - Entomologische Nachrichten und Berichte 49 (3/4): 185-189.

Klausnitzer, B. 2009a: Neue Arten der Gattung Prionocyphon L. Redtenbacher, 1858 aus Nepal (Insecta: Coleoptera: Scirtidae). - In: Hartmann, M. \& Weipert, J. (Hrsg.): Biodiversität und Naturausstattung im Himalaya III. - Verein der Freunde \& Förderer des Naturkundemuseums Erfurt e. V.: 295-297, Tafel X + XI.

Klausnitzer, B. 2009b: Untergattungen bei Prionocyphon L. RedTEnbacher, 1858 (Coleoptera, Scirtidae). - Entomologische Nachrichten und Berichte 53 (2): 105-114.

Klausnitzer, B. 2010: Neue Arten von Mescirtes Motschulsky, 1863 aus Vietnam (Coleoptera, Scirtidae). - Denisia 29: 165-172.

Klausnitzer, B. 2012a: Neue Arten von Mescirtes Motschulsky, 1863 aus Südostasien (Coleoptera: Scirtidae). - Entomologische Zeitschrift 122 (2): 69-74.

Klausnitzer, B. 2012b: Zur Kenntnis der Gattung Mescirtes Motschulsky, 1863 (Coleoptera, Scirtidae). - Entomologische Blätter und Coleoptera 108: 201-207.

Klausnitzer, B. 2013: Eine neue Art der Gattung Mescirtes Mотschulsky, 1863 aus China (Coleoptera, Scirtidae). - Entomologische Nachrichten und Berichte 57 (3): 127-130.

RutA, R. 2009: Revision of Scirtidae (Insecta: Coleoptera) described by Victor Ivanovitsch Motschulsky. Zootaxa 2210: 26-50. 
Ruta, R. 2010: Two new species of Prionocyphon RedTENBACHer from Taiwan (Coleoptera: Scirtidae), with notes on Prionocyphon, Mescirtes Motschulsky and Prionoscirtes CHAMPION from East and Southeast Asia. - Zootaxa 2402: 52-60.
Yoshitomi, H. \& SAtô, M. 2003: Scirtidae of the Oriental Region, Part 3. A New Species of the Genus Prionocyphon (Coleoptera: Scirtidae) from Laos, with a World Check List of the Species. - Entomological Review of Japan 58: 187-190.
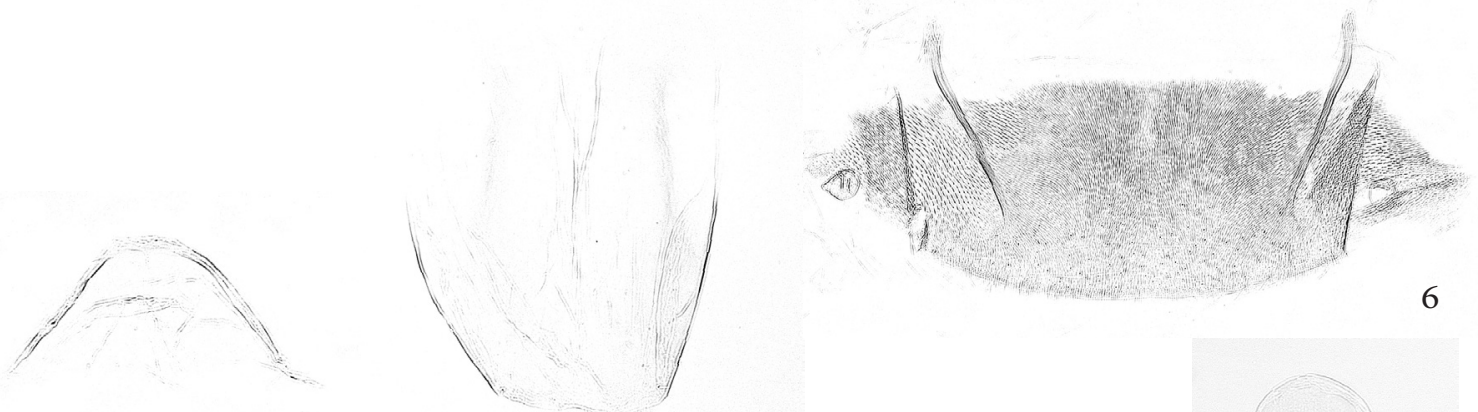

4

5
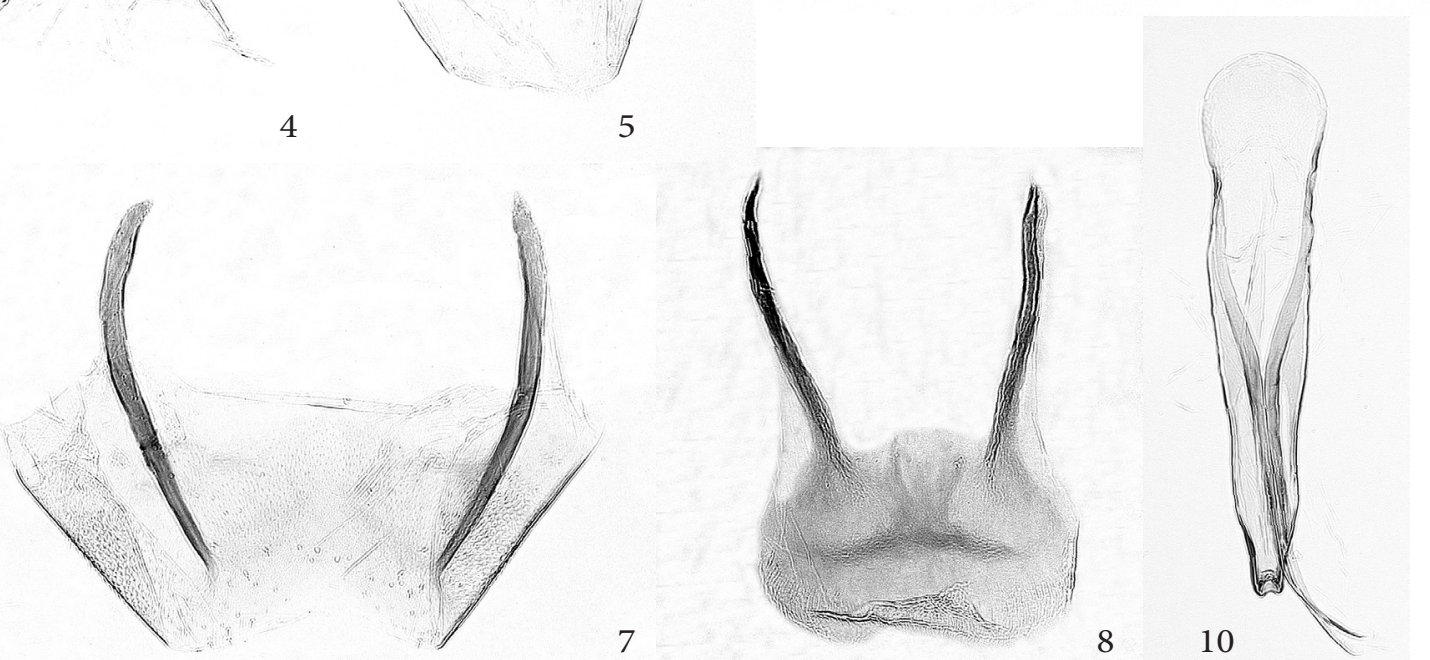

8

10
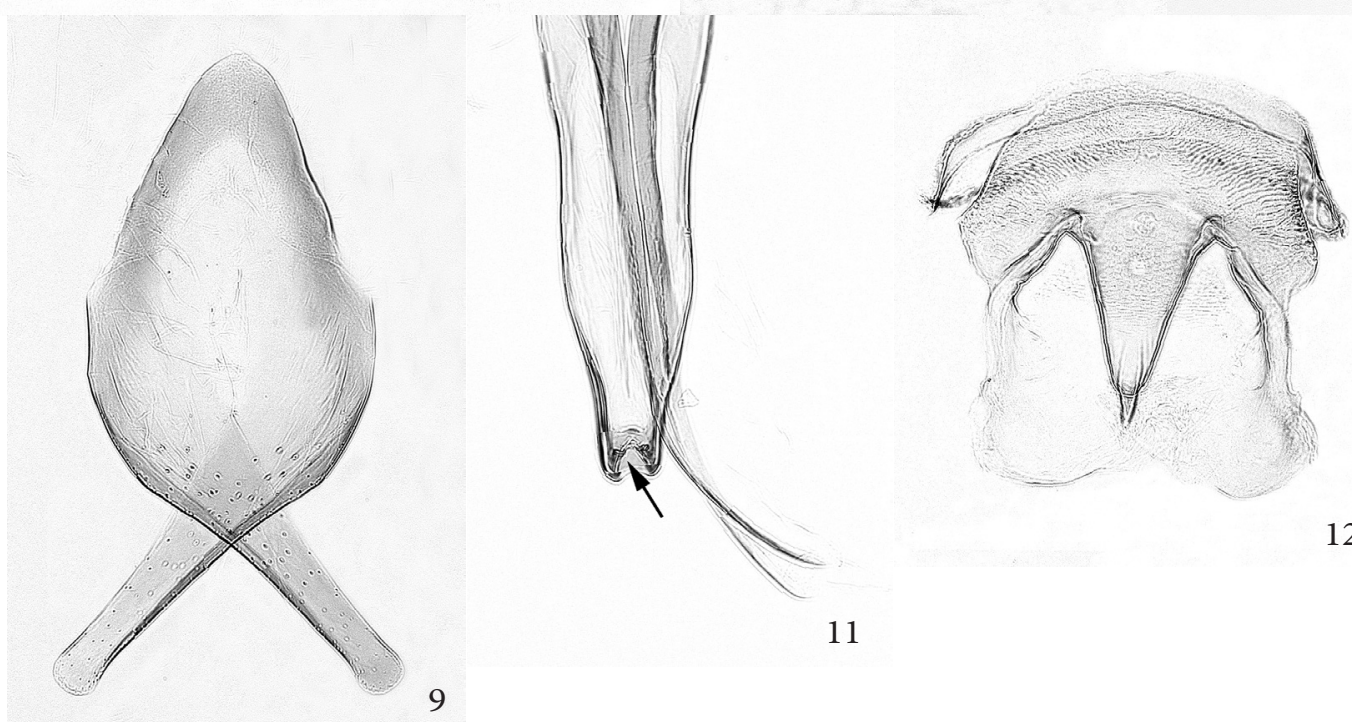

12

Fig. 4-12: Mescirtes sedlagi n. sp. (4) 8. Sternit (5) Mescirtes sedlagi n. sp., 9. Sternit (6) Mescirtes sedlagi n. sp., 7. Tergit (7) Mescirtes sedlagi n. sp., 8. Tergit (8) Mescirtes sedlagi n. sp., 9. Tergit (9) Mescirtes sedlagi n. sp., Tegmen (artifiziell in der Mitte überkreuzt) (10) Mescirtes sedlagi n. sp., Penis (11) Mescirtes sedlagi n. sp., Penis, Trigonium und Parameroide (12) Mescirtes sedlagi n. sp., ㅇ, Bursalsklerit. 


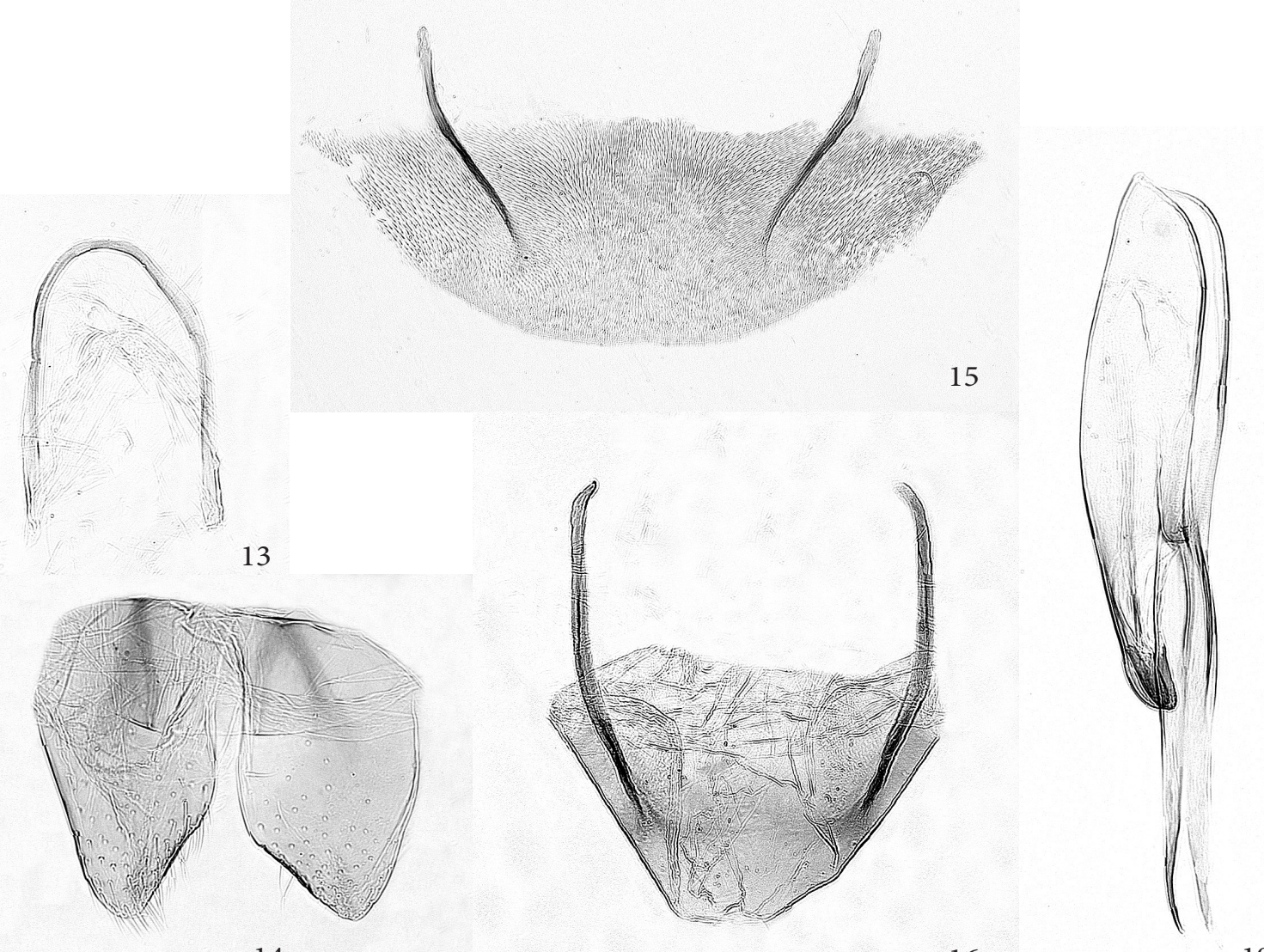

14

16

19

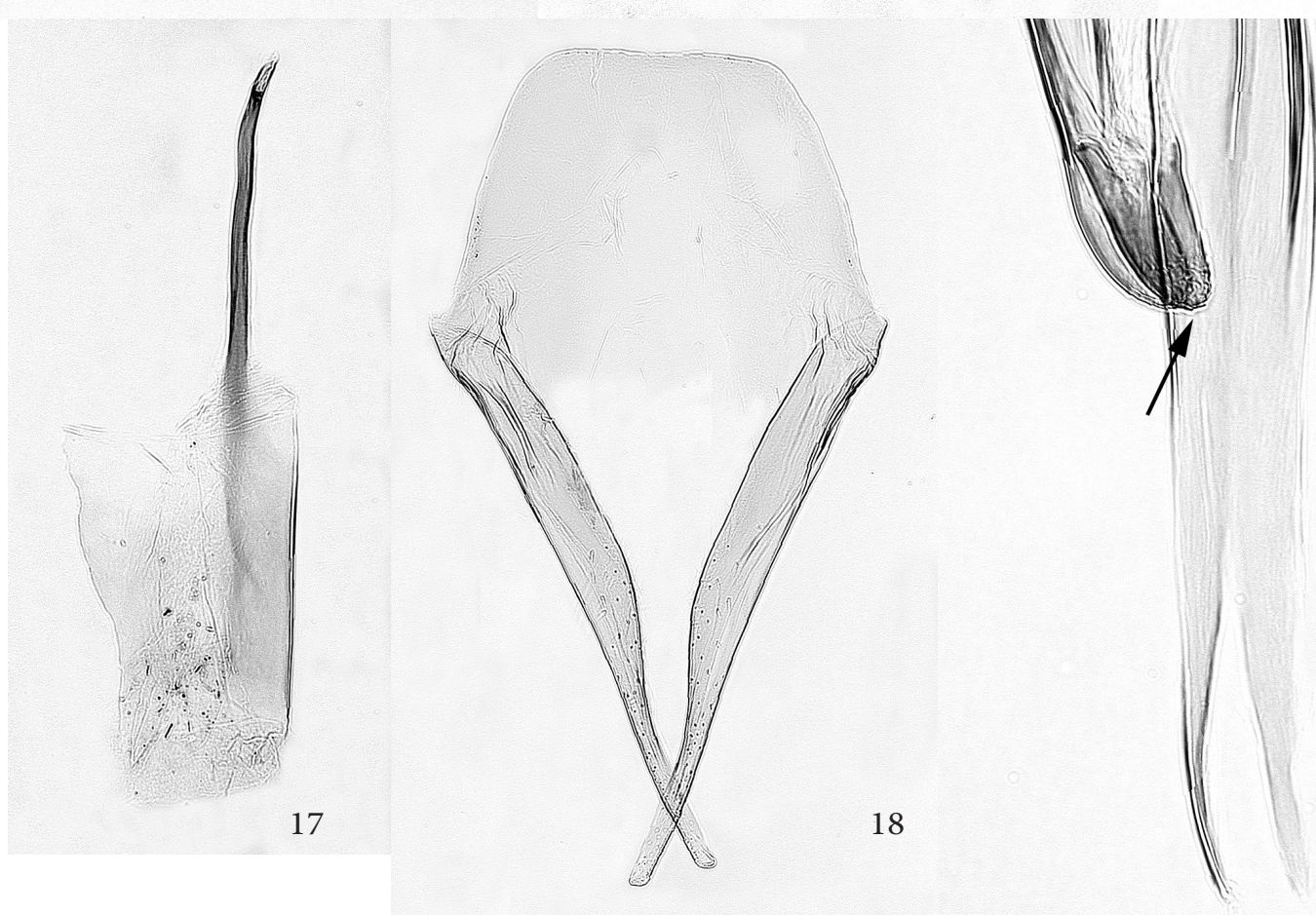

20

Fig. 13: Mescirtes eberti n. sp., 8. Sternit. - Fig. 14: Mescirtes eberti n. sp., 9. Sternit. - Fig. 15: Mescirtes eberti n. sp., 7. Tergit. Fig. 16: Mescirtes eberti n. sp., 8. Tergit. - Fig. 17: Mescirtes eberti n. sp., 9. Tergit (Hälfte). - Fig. 18: Mescirtes eberti n. sp., Tegmen. Fig. 19: Mescirtes eberti n. sp., Penis. - Fig. 20: Mescirtes eberti n. sp., Penis, Trigonium und Parameroide. 